\title{
Plan de cuidados de enfermería para la prevención de úlceras por presión secundarias a la posición prono en pacientes COVID-19
}

Nursing care plan for the prevention of pressure ulcers secondary to prone positioning in COVID-19 patients

Plano de cuidados de enfermagem para a prevenção de úlceras por pressão secundárias à posição prona em pacientes com COVID-19

Cómo citar este artículo:

Rojas LZ, Mora Rico LA, Acosta Barón JV, Cristancho Zambrano LY, Valencia Barón YD, Hernández Vargas JA. Plan de cuidados de enfermería para la prevención de úlceras por presión secundarias a la posición prono en pacientes COVID-19. Revista Cuidarte. 2021;12(3):e2234. http://dx.doi.org/10.15649/cuidarte.2234

Revista Cuidarte

Rev Cuid. Sep - Dic 2021; 12 (3): e2234 doij http://dx.doi.org/10.15649/cuidarte.2234

E-ISSN: 2346-3414

(1) Lyda Z. Rojas ${ }^{1}$

(1) Liliana Andrea Mora Rico ${ }^{2}$

(1) Jennifer Vanesa Acosta Barón ${ }^{3}$

(1) Luisa Yaneth Cristancho Zambrano ${ }^{4}$

(1) Yurley Dayanna Valencia Barón ${ }^{5}$

(1) Juliana Alexandra Hernández Vargas ${ }^{6}$

1 Fundación Cardiovascular de Colombia, Grupo de Investigación y Desarrollo de Conocimiento en Enfermería GIDCEN, Floridablanca, Colombia. Email: lydarojas@fcv.org Autor de Correspondencia.

2 Hospital Internacional de Colombia, Piedecuesta, Colombia. Email: lillianamora@fcv.org

3 Hospital Internacional de Colombia, Piedecuesta, Colombia. Email: jenniferacosta@fcv.org

4 Hospital Internacional de Colombia, Piedecuesta, Colombia. Email: luisacristancho@fcv.org

5 Fundación Cardiovascular de Colombia, Centro de Investigaciones, Floridablanca, Colombia. Email: yurleyvalencia@fcv.org

6 Cuenta de Alto Costo, Fondo Colombiano de Enfermedades de Alto Costo, Bogotá, Colombia. Grupo de Investigación y Desarrollo de Conocimiento en Enfermería GIDCEN. Email:

jhernandez@cuentadealtocosto.org

\section{Resumen}

Introducción: La posición prono (PP) es una alternativa terapéutica ampliamente recomendada e implementada en los pacientes con COVID-19. Sin embargo, aunque es un procedimiento no invasivo, es complejo y se asocia con eventos adversos como las úlceras por presión (UPP). Nuestro objetivo es proponer un plan de cuidados de enfermería basado en el lenguaje estandarizado NANDA-I, NIC, NOC para la prevención de las UPP secundarias a la PP en la enfermedad de COVID-19. Síntesis del contenido: En los pacientes con COVID-19, además de factores de riesgo propios del paciente como la edad avanzada y la presencia de comorbilidades, la PP contribuye a la presencia de los diagnósticos de enfermería de riesgo de úlcera por presión [00249], de deterioro de la integridad cutánea [00047] y tisular [00248]. Por su parte, la intervención de enfermería prevención de úlceras por presión [3540], es clave para minimizar el desarrollo de esta complicación, mejorar la calidad de la atención y el pronóstico en este tipo de pacientes. Finalmente, para determinar la efectividad del cuidado de enfermería se proponen los resultados NOC consecuencias de la inmovilidad: fisiológicas [0204] e integridad tisular: piel y membranas mucosas [1101]. Conclusión: La PP es una terapia coadyuvante recomendada para el manejo de los pacientes con COVID-19 críticamente enfermos, debido a que optimiza la función pulmonar, sin embargo está asociada a eventos adversos como las UPP. Este artículo presenta recomendaciones basadas en una revisión narrativa para facilitar la implementación de cuidados de enfermería preventivos que reduzcan su frecuencia en esta población.

Palabras clave: Prevención \& control, Úlcera por presión, Posición prona, Infecciones por Coronavirus, Pandemias.

Recibido: 30 de abril de 2021

Aceptado: 02 de agosto de 2021

Publicado: 13 de septiembre de 2021 $\square *$ Correspondencia

Lyda Z. Rojas

E-mail: lydarojas@fcv.org 


\section{Nursing care plan for the prevention of pressure ulcers secondary to prone positioning in COVID-19 patients}

\section{Abstract}

Introduction: Prone position (PP) is a therapeutic alternative widely used and recommended in patients with COVID-19. Although PP is a non-invasive procedure, it is complex and could be associated with complications such as the development of pressure ulcers (PU). We aimed to propose a standardized nursing care plan in terms of NANDA-International, NIC (Nursing Interventions Classification) and NOC (Nursing Outcomes Classification) to prevent PU secondary to the PP in people with COVID-19. Content synthesis: In patients with COVID-19, in addition to risk factors such as advanced age and the presence of comorbidities, PP contributes to the presence of pressure ulcer risk nursing diagnoses [00249], of deterioration of skin [00047] and tissue [00248] integrity. On the other hand, the nursing intervention for the prevention of pressure ulcers [3540], due to the specificity and scientific basis of its activities, is key to minimize the development of this complication, improve the quality of care and the prognosis in this type of patients. Finally, to evaluate the effectiveness of nursing care, we propose the nursing results (NOC): consequences of immobility: physiological [0204] and tissue integrity: skin and mucous membranes [1101]. Conclusion: PP is a recommended adjunctive therapy for the management of critically ill COVID-19 patients due to its benefits to improve lung function. However, it is associated with adverse effects such as PU. This article presents recommendations based on a narrative review for a better implementation of preventive nursing care that reduces the frequency of PU in this population.

Keywords: Prevention \& control, Pressure Ulcer, Prone Position, Coronavirus infections, Pandemics.

\section{Plano de cuidados de enfermagem para a prevenção de úlceras por pressão secundárias à posição prona em pacientes com COVID-19}

\section{Resumo}

Introdução: A posição prona (PP) é uma alternativa terapéutica amplamente recomendada e implementada em pacientes com COVID-19. No entanto, embora seja um procedimento não invasivo, é complexo e está associado a eventos adversos, como úlceras por pressão (UP). Nosso objetivo é propor um plano de cuidados de enfermagem baseado na linguagem padronizada NANDA-I, NIC, NOC para a prevenção de UP secundárias à PP na doença COVID-19. Síntese de conteúdo: Em pacientes com COVID-19, além dos próprios fatores de risco do paciente, como idade avançada e presença de comorbidades, a PP contribui para a presença de diagnósticos de enfermagem de risco de úlcera por pressão [00249], de deterioração da pele [00047] e do tecido Integridade. Por sua vez, a intervenção de enfermagem na prevenção de úlceras por pressão [3540] é fundamental para minimizar o desenvolvimento desta complicação, melhorar a qualidade da assistência e o prognóstico neste tipo de paciente. Por fim, para determinar a eficácia da assistência de enfermagem, são propostos os resultados da NOC, consequências da imobilidade: fisiológicas [0204] e integridade do tecido: pele e mucosas [1101]. Conclusão: PP é uma terapia adjuvante recomendada para o tratamento de pacientes graves com COVID-19, pois otimiza a função pulmonar, porém está associada a eventos adversos, como UP. Este artigo apresenta recomendações baseadas em revisão narrativa para facilitar a implementação de cuidados preventivos de enfermagem que reduzam sua frequência nesta população.

Palavras chave: Prevencão \& controle, Lesão por Pressão, Decúbito Ventral, Infecções por coronavirus, Pandemias. 


\section{Introducción}

La característica principal de la enfermedad grave por coronavirus 2019 (COVID-19) es la lesión pulmonar aguda manifestada en el síndrome de dificultad respiratoria aguda (SDRA) ${ }^{1}$ cuya prevalencia oscila entre el $20-41 \%^{2}$. La posición prono (PP) se indica entre el $16 \%{ }^{3}$ y el $33 \%{ }^{4}$ de las personas con SDRA para manejar la lesión pulmonar asociada a la ventilación y promover la oxigenación en los casos graves ${ }^{1,5,6}$. En la PP el paciente está acostado horizontalmente con la parte frontal del cuerpo hacia abajo y la cabeza ubicada neutralmente; es diferente de la pronación (es decir, la rotación del antebrazo de manera que la palma de la mano quede hacia abajo) y opuesta a la posición supina7.

La PP está indicada en cirugías de columna, cuello, neurocirugía, colorrectales, vasculares y más recientemente, para el manejo de los pacientes con COVID-195. Los principales mecanismos por los que la PP mejora la condición de los pacientes con SDRA son: mejorar la captación en las regiones pulmonares dorsales, aumentar el volumen pulmonar al final de la espiración y la elasticidad de la pared torácica, disminuir el shunt alveolar y optimizar el volumen corriente ${ }^{8}$.

Según los resultados de dos metaanálisis, la PP se asoció con una disminución de la mortalidad, aunque no fue estadísticamente significativa ${ }^{9,10}$, sin embargo, en los análisis de subgrupos se evidenció que la PP disminuyó el riesgo de morir en el $42 \%$ de los pacientes ventilados con volumen corriente bajo; de éstos, el $40 \%$ se encontraba en pronación prolongada, el $51 \%$ en instauración de la PP antes de las 48 horas de evolución de la enfermedad y el $49 \%$ en hipoxemia 9 . Asimismo, redujo la mortalidad en $26 \%$ en los pacientes con 12 horas o más de duración de la PP y en aquellos con SDRA moderado a severo y la relación $\mathrm{PaO}_{2} / \mathrm{FiO}_{2}$ al día cuarto fue significativamente mayor en el grupo de PP en comparación con el de posición supina (diferencia de medias: 23,5; IC 95\% 12,4 a 34,5) ${ }^{10}$.

Debido a estos beneficios y a la buena respuesta a la ventilación en los pacientes críticos en PP, su uso se ha incrementado como alternativa terapéutica en el manejo del COVID-1911, Ilevando a que sea recomendada internacionalmente en las guías de práctica clínica de cuidados intensivos ${ }^{12,13}$.

Sin embargo, la PP tiene algunos aspectos a considerar para su implementación. Aunque es un procedimiento no invasivo, es complejo y puede generar importantes eventos adversos que requieren de equipos capacitados para su prevención. Adicionalmente, es una tarea agotadora y demandante para el personal de las unidades de cuidados intensivos sometido a condiciones de estrés ${ }^{6}$, que sumadas a las particularidades de la pandemia, como la incertidumbre constante sobre los recursos, las capacidades y los riesgos que enfrentan los trabajadores de la salud, así como la exposición al sufrimiento, la muerte y las amenazas a su propia seguridad, incrementan la carga laboral, especialmente del personal de enfermería ${ }^{14}$.

Por otra parte, la PP se asocia con complicaciones como el desarrollo de úlceras por presión (UPP), siendo el evento adverso más frecuente (34\%), seguido de la neumonía asociada a la ventilación mecánica (21\%), la obstrucción del tubo orotraqueal (15\%), la extubación accidental (11\%), la pérdida del acceso venoso (11\%), el neumotórax (6\%) y el desplazamiento

\author{
La PP se asocia con \\ complicaciones como el \\ desarrollo de úlceras por presión \\ (UPP), siendo el evento adverso \\ más frecuente (34\%)
}


del tubo orotraqueal (4\%) ${ }^{9}$. Particularmente, en los pacientes con COVID-19 ventilados en PP, la prevalencia de las UPP oscila entre el $48 \%{ }^{15}$ y el $61 \%{ }^{16}$. En términos de su localización, se observó una mayor frecuencia en el mentón, la frente, los pómulos y la nariz ${ }^{16}$ y en cuanto a su severidad, las lesiones grado II son las más comunes $(\sim 64 \%)^{17,18}$.

Se han descrito diversos factores asociados con el desarrollo de UPP secundarias a la PP en comparación con la posición supina, los cuales incrementan hasta 3,3 veces el riesgo de UPP, con mayor afectación del área facial como se expuso previamente ${ }^{19-22}$. Estos factores son similares a los descritos en otras poblaciones críticamente enfermas que requieren PP dentro del plan de manejo, incluyendo: a) la inmovilidad, la mala percepción y respuesta sensorial y, b) la susceptibilidad y tolerancia individual donde las características de la piel, la pobre perfusión y nutrición, la diabetes, el exceso de humedad y la albúmina disminuida son factores contribuyentes $^{23}$. Además de lo anterior, un claro factor de riesgo es el promedio de duración de la PP, que se relaciona directamente con la aparición y la severidad de las UPP en las personas con COVID-19 15-17, incrementando sustancialmente el riesgo, incluso desde el tercer día ${ }^{15}$.

En relación con el tratamiento de las UPP, este es largo y genera una carga económica importante para el sistema de salud. En los Estados Unidos, se gastan aproximadamente \$11 mil millones de dólares anuales derivados de su cuidado y, entre $\$ 500$ y $\$ 70.000$ en una sola herida ${ }^{24}$. Aunque no se ha realizado una estimación del incremento en los costos asociados al cuidado de las UPP en los pacientes con COVID-19 se hipotetiza un impacto importante, a tal punto que el cuidado de las heridas ha cobrado mayor relevancia, pasando de ser un área subestimada a un eje clave dentro de las guías y protocolos de atención, incluyendo las relacionadas con los cuidados de enfermería.

En este sentido, además del control de los síntomas y el tratamiento de las complicaciones secundarias a la COVID-19, se deben implementar medidas preventivas frente a las UPP derivadas de la $\mathrm{PP}^{21}$, con el fin de disminuir la alta tasa de incidencia de este evento adverso producto de la atención en salud de los pacientes con SDRA.

En consideración con lo expuesto, el objetivo de este artículo es proponer un plan de cuidados de enfermería basado en los lineamientos establecidos en NANDA Internacional (NANDA-I), NIC (Nursing Interventions Classification) y NOC (Nursing Outcomes Classification) para la prevención de las UPP secundarias a la PP en la enfermedad de COVID-19, con el fin de orientar la implementación y evaluación de intervenciones basadas en una revisión narrativa que contribuyan a una mayor

El objetivo de este artículo es proponer un plan de cuidados de enfermería basado en los lineamientos establecidos en NANDA Internacional (NANDA-I), NIC (Nursing Interventions Classification) y NOC (Nursing Outcomes Classification) para la prevención de las UPP secundarias a la PP en la enfermedad de COVID-19 seguridad y calidad de la atención, teniendo en cuenta que las UPP se consideran un evento adverso producto de fallas en la atención de enfermería.

\section{Factores de riesgo y diagnósticos de enfermería en las UPP}

Los pacientes con COVID-19 hospitalizados en unidades de cuidado crítico presentan factores de riesgo que favorecen el desarrollo de las UPP como los extremos de la vida (edad avanzada 
$60,5 \pm 14,5$ años), el estado nutricional desequilibrado (sobrepeso/obesidad, con una mediana del índice de masa corporal de 30,5, Q1=26,6; Q3=36,2), los agentes químicos/secreciones (náuseas o vomito 17,7\%, diarrea 20,8\%), las comorbilidades (hipertensión 59,7\%, diabetes $38,9 \%$, enfermedades pulmonares crónicas 24,0\%) y la hipoperfusión (mediana $\mathrm{PaO}_{2} / \mathrm{FiO}_{2}$ 124, Q1=86; Q3=188) generada por la disnea (74,9\%), la fatiga $(32,5 \%)$ o el tratamiento con vasopresores $(48,3 \%)^{25}$.

De igual forma, los casos graves tienen menores niveles de hemoglobina comparados con los moderados (diferencia de medias ponderada de hemoglobina -4,08 g/L; IC del 95\% -5, 12;-3,05) ${ }^{26}$ y fricción con la superficie/disminución de la movilidad (posición prono 27,0\% y 38,5\%) ${ }^{25,27}$ así como hipertermia (fiebre mediana $\left.38,1^{\circ} \mathrm{C}, \mathrm{Q} 1=37,3 ; \mathrm{Q} 3=38,9\right)^{25}$.

Teniendo en cuenta estas características y la coincidencia de estos factores de riesgo con los enunciados en NANDA-I, se proponen tres diagnósticos de enfermería para prevenir las UPP secundarias a la PP en la enfermedad de COVID-19: "riesgo deterioro de la integridad cutánea [00047]" definido como la susceptibilidad a una alteración de la epidermis y/o de la dermis que puede comprometer la salud, "riesgo de deterioro de la integridad tisular [00248]" definido como susceptible a una lesión de la membrana mucosa, córnea, sistema intertegumentario, fascia muscular, músculo, tendón, hueso, cartílago, cápsula de la articulación y/o ligamento que puede comprometer la salud y, el "riesgo de úlcera por presión [00249]" definido como la susceptibilidad a lesiones localizadas de la piel y/o tejido subyacente por lo general en un relieve óseo como resultado de la presión, o la presión en combinación con el cizallamiento ${ }^{28}$.

\section{Cuidados de enfermería para la prevención de las UPP}

Para la selección de las intervenciones y los resultados de enfermería se tuvo en cuenta los vínculos entre las clasificaciones NANDA-I (2018-2020), NOC (6a edición) y NIC (7a edición), identificados con la herramienta NNNConsult ${ }^{28}$. Se priorizó la intervención "Prevención de úlceras por presión [3540]" definida como la prevención de la formación de UPP en un individuo con alto riesgo de desarrollarlas, dada su robustez para atender los factores de riesgo identificados en los tres diagnósticos de enfermería previamente propuestos y el alcance general de las actividades que sugiere. Para fundamentar las actividades descritas en dicha intervención se realizó una revisión narrativa, para lo cual se hicieron búsquedas en las bases de datos PUBMED, EMBASE y CINALH, combinando los siguientes términos libres: "Pressure Ulcers " OR " Pressure Sore " OR " Pressure Injuries " AND " Prone Position " AND " COVID-19". Se seleccionaron los artículos que permitieron justificar las actividades seleccionadas (Tabla 1).
Se priorizó la intervención "Prevención de úlceras por presión [3540]" definida como la prevención de la formación de UPP en un individuo con alto riesgo de desarrollarlas, dada su robustez para atender los factores de riesgo identificados en los tres diagnósticos de enfermería previamente propuestos y el alcance general de las actividades que sugiere. 


\section{Tabla 1. Intervención de enfermería: Prevención de úlceras por presión (3540)}

Actividades

(NIC) 28

\section{Referencia}

La evaluación del riesgo de UPP debe efectuarse antes que el paciente sea candidato para la PP y cuando retorne a la posición de supino según el plan de cuidados ${ }^{29}$.

La evaluación del riesgo de UPP se considera una buena práctica de cuidado. No obstante, la frecuencia con la que debe realizarse no está bien definida; depende del juicio clínico y el riesgo inherente al paciente, dado por el pronóstico clínico y el número de dispositivos de cuidado instalados ${ }^{30}$.

Utilizar una

herramienta de

valoración de riesgo

establecida para

valorar los factores

de riesgo del

individuo (escala de Braden).

No se ha descrito una escala específica para evaluar el riesgo de UPP en esta población. Sin embargo, los resultados de un meta-análisis sugieren que las escalas de Braden, Norton y Waterlow tienen una sensibilidad, especificidad y valores predictivos similares y pueden aplicarse en contextos de cuidado crítico ${ }^{31,32}$.

En los casos en los que se observe la úlcera por presión, la valoración del riesgo debe continuarse con el Sistema Internacional de Clasificación de las Úlceras por Presión del Panel Asesor Nacional de UPP (NPIAP, por su sigla en inglés) y del Panel Asesor Europeo de UPP (EPUAP, por su sigla en inglés) $)^{33}$.

Se recomienda realizar más de dos evaluaciones del riesgo de UPP al día en aquellos pacientes con múltiples dispositivos médicos, cambios de líquidos frecuentes y con signos de edema localizado o generalizado ${ }^{33}$.

Registrar el estado de la piel durante el ingreso y luego a diario.

La vigilancia de las características de la piel, debe incluir la valoración de las mucosas, puesto que el eritema no blanqueable es poco visible. Es importante realizar una inspección detallada de la mucosa oral debido a que lesiones aparentemente superficiales pueden ser más profundas y estar asociadas a una mayor extensión ${ }^{33}$

Eliminar la humedad excesiva en la piel causada por la transpiración, el drenaje de heridas y

la incontinencia

fecal o urinaria.

Vigilar las fuentes de presión y de fricción.

Es necesario mantener la piel limpia e hidratada en un nivel óptimo, evitando el exceso de humedad o resequedad que pueden generar maceración ${ }^{30}$.

La vigilancia del nivel de hidratación de la piel es fundamental debido a que la mayoría de pacientes con SDRA son llevados a un balance hídrico negativo ${ }^{33}$.

La frecuencia de limpieza de la piel debe ser determinada de manera individual para no afectar la hidratación de la piel que tiene una función de barrera natural contra las consecuencias de la fricción ${ }^{33}$.

La cara y aquellas áreas corporales que soportan mayor peso como la región mamaria, el tórax, las clavículas, la cresta ilíaca y las rodillas han sido descritas en la literatura como las de mayor

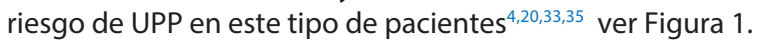

La frente, los pómulos y la barbilla son las zonas más susceptibles al desarrollo de UPP en los pacientes sometidos a la PP. Por lo anterior, se debe extremar su valoración periódica y la liberación de las fuentes de presión o fricción ${ }^{36}$.

En los hombres, es particularmente importante vigilar que no existan zonas de presión en el área genital $^{35}$.

Inspeccionar la piel de las prominencias óseas y demás puntos de presión al cambiar de posición al menos una vez al día.

La presencia de dispositivos médicos (circuitos de infusión, monitorización de signos vitales o sondajes) es un factor de riesgo para los pacientes en la PP debido a la presión sostenida que ejercen sobre ciertas zonas del cuerpo, por lo que se debe evitar el contacto estrecho con los $\operatorname{mismos}^{33,37}$.

En las personas con tubos endotraqueales, los sitios anatómicos que se han asociado con una mayor frecuencia de UPP son los labios y la boca ${ }^{35,38}$.

En las personas con sondas nasogástricas, la valoración de la nariz, las narinas y el puente nasal es fundamental debido a que estas lesiones son más frecuentes en dichos sitios anatómicos ${ }^{35,38}$.

Evitar el agua caliente y utilizar un jabón suave para el baño.
Mantener la piel limpia e hidratada utilizando productos con $\mathrm{pH}$ balanceado es prioritario. Sin embargo, la evidencia sobre el tipo de hidratantes que se deben emplear es inconsistente ${ }^{29}$.

Tradicionalmente, la limpieza de la piel se ha realizado con agua y jabón. No obstante, existe evidencia de su impacto desfavorable sobre el balance del $\mathrm{pH}$. Al respecto, otros productos que contienen surfactantes pueden proveer una mayor protección a la piel ${ }^{33,39,40}$.
Aplicar barreras de protección, como cremas o compresas absorbentes, para eliminar el exceso de humedad, según corresponda.
El uso de limpiadores con pH entre 4,0 y 7,0 (ligeramente ácido a neutro) ha sido costoefectivo para reducir la resequedad, el eritema, la irritación de la piel y, en general la incidencia de las UPP $33,39,40$.

La aplicación de ácidos grasos hiperoxigenados es efectiva en la prevención de las UPP relacionadas con la ventilación mecánica en este grupo de pacientes ${ }^{41}$.

El uso profiláctico de apósitos como hidrocoloides, películas transparentes y de silicona han sido eficaces para disminuir la presión y la degradación de la piel de la cara, principalmente ${ }^{19,29}$ ver Figura 2.

El uso de hidrocoloides y películas transparentes reduce hasta en un 50\% la incidencia de las UPP en las áreas faciales ${ }^{42}$. 


\section{Actividades} (NIC) 28

\section{Referencia}

Colocar al paciente en posición ayudándose con almohadas para elevar los puntos de presión encima del colchón.

Utilizar mecanismos en la cama (badana) para proteger al paciente.

Evitar mecanismos de tipo flotador para la zona sacra.
El uso profiláctico de apósitos en las zonas circundantes a la instalación de dispositivos médicos como el circuito de ventilación mecánica, sondas nasogástricas o sistemas de infusión de medicamentos es recomendable para disminuir la presión y la fricción ${ }^{5,30,33,38}$.

Teniendo en cuenta que la presencia de tubos endotraqueales y sondas nasogástricas incrementa el riesgo de UPP, su reposicionamiento lateral ha demostrado ser beneficioso para la prevención de lesiones de tejidos blandos ${ }^{33}$.

El uso de superficies de apoyo adecuadas y almohadas es crucial para prevenir la deformación del tejido y mejorar la perfusión tisular ${ }^{43}$.

El uso de sistemas de soporte para la cabeza en la PP reduce significativamente la incidencia de las UPP ${ }^{43}$.

Con respecto a los dispositivos para dar soporte a la cabeza, aquellos en forma de anillo o dona deben evitarse debido a que causan un mayor cizallamiento y presión en la superficie y capas internas de la piel, incrementando el riesgo de UPP ${ }^{44,45}$

Con respecto a los dispositivos para dar soporte a la cabeza, aquellos en forma de anillo o dona deben evitarse debido a que causan un mayor cizallamiento y presión en la superficie y capas internas de la piel, incrementando el riesgo de UPP ${ }^{44,45}$.

El uso de almohadas para el acojinamiento de las estructuras faciales ha sido eficiente para disminuir la incidencia de las UPP ${ }^{46}$.

El desplazamiento y la deformidad de la piel y los tejidos blandos no dependen de la dureza de los colchones sino de su forma. Una mayor inclinación del colchón incrementa el desplazamiento de la superficie de la piel y el riesgo de UPP ${ }^{47}$.

Como parte del cuidado de enfermería, es prioritario identificar las actividades específicas para ciertas áreas corporales expuestas a un alto riesgo:

- Ocular: además de la lesión corneal, la PP puede ocasionar pérdida de la visión debida principalmente a isquemia del nervio óptico o síndrome compartimental. Para su prevención se recomienda el uso de reposacabezas para liberar la presión directa sobre las estructuras oculares ${ }^{18,46,48}$

La aplicación de lubricante y cerrar los párpados con cinta adhesiva también es recomendable ${ }^{18}$.

Adicionalmente, la posición de la cama en Trendelenburg es útil para reducir la presión ocular y el edema ${ }^{48}$.

- Tórax: los cables de los dispositivos de monitoreo hemodinámico o de los sistemas de infusión deben reposicionarse en la zona de la espalda o lateralmente en las extremidades con el fin de evitar su presión excesiva.

Se debe garantizar que todas las líneas centrales, arteriales o cánulas estén perfectamente aseguradas y en lo posible, fijadas lejos del área del tórax ${ }^{18}$.

- Genitales y extremidades inferiores: Las sondas vesicales o dispositivos para la eliminación fecal deben posicionarse fuera de la zona que está en contacto estrecho con la cama ${ }^{18}$.

Se recomienda el uso de apósitos profilácticos o férulas en la patela o el área pretibial ${ }^{18}$.

El uso de dispositivos para mejorar la posición de las zonas de alto riesgo, sumado a los cambios de posición son altamente recomendables para disminuir los puntos de presión en la cara y otras áreas corporales ${ }^{29}$.

Las superficies de soporte funcionan para redistribuir la presión y el cizallamiento en la piel, promoviendo una mejor perfusión tisular y aliviando las deformaciones del tejido ${ }^{49,50}$.

Darse la vuelta continu- Los cambios de posición habituales con una frecuencia determinada según la evaluación del riesgo, amente cada 1-2 horas, según corresponda. así como el reposicionamiento de los dispositivos médicos son eficientes para disminuir las zonas de presión y evitar la ruptura de la piel y los tejidos blandos ${ }^{33}$.

El reposicionamiento corporal debe realizarse como mínimo cada dos horas en los pacientes que han sido sometidos a ciclos de 12 horas o más en esta posición ${ }^{5,35}$.

Darse la vuelta con cuidado (p. ej., evitar el cizallamiento) para evitar lesiones en una piel frágil.

Poner el programa de cambios posturales al lado de la cama, según corresponda.
Al realizar los cambios de posición es fundamental conservar una buena alineación esquelética con el fin de reducir la tensión muscular a un nivel mínimo. EI NPIAP recomienda la posición de natación utilizando almohadas para dar soporte a las zonas corporales, alternando la posición de los brazos y la cabeza cada 2 horas para disminuir el riesgo de UPP en los codos y la cara (Figura 3). Se debe garantizar que el tubo endotraqueal no se desplace u obstruya durante el reposicionamiento ${ }^{5,18,51}$.

NIC: Nursing Intervention Classification.

\section{Evolución y eficiencia de los cuidados preventivos de las UPP}

Para la selección de los resultados se tuvo en cuenta la población del contexto del artículo (pacientes críticos con SDRA secundaria a COVID-19 en posición prona, en unidades de 
cuidados intensivos y sedados) ${ }^{52}$ y los vínculos NANDA-I, NOC y NIC. Por tanto, sugerimos dos resultados para monitorizar la efectividad de las intervenciones y evitar que se avance a diagnósticos reales de enfermería. El primero, es la "Integridad tisular: piel y membranas mucosas [1101]" definido como la indemnidad estructural y función fisiológica normal de la piel y las membranas mucosas, con los indicadores de: hidratación [110104], perfusión tisular [110111], integridad de la piel [1 10113] medidos con la escala 01 (Grado de deterioro de la salud o el bienestar) y los indicadores lesiones de la mucosa [110116], eritema [1101121], palidez [110122] evaluados con la escala 14 (Grado de un estado o respuesta negativo a adverso) con opciones de respuesta de grave (1), sustancial (2), moderado (3), leve (4) y ninguno (5) ${ }^{28}$. El segundo se denomina "Consecuencias de la inmovilidad: fisiológicas [0204]", definido como la gravedad del compromiso en el funcionamiento fisiológico debido a la alteración de la movilidad física y su indicador más pertinente es el de úlceras por presión [20401] ${ }^{28}$.

En la Tabla 2 se observa un ejemplo de la operacionalización del indicador úlceras por presión [20401] ${ }^{28}$ utilizando las escalas de evaluación del riesgo de UPP. Cada parámetro evaluado califica del 1 al 4 (siendo 1 el peor estado 1 y 4 el mejor) y en términos de los resultados de enfermería (Grave:1, Moderado:2, Leve:3 y Ninguno:4), arbitrariamente omitimos la categoría "sustancial" para que coincida con las escalas de medición de los instrumentos para evaluar el riesgo de UPP y así no alterar sus propiedades En la Tabla 2 se observa un ejemplo de la operacionalización del indicador úlceras por presión [20401] $]^{28}$ utilizando las escalas de evaluación del riesgo de UPP. psicométricas.

Tabla 2. Operacionalización del indicador úlceras por presión [20401] utilizando las escalas de evaluación del riesgo

Consecuencias de la inmovilidad: fisiológicas [0204](28)

\begin{tabular}{|c|c|c|c|c|c|}
\hline $\begin{array}{l}\text { Indicador } \\
\text { NOC }\end{array}$ & Parámetro a evaluar & Grave (1) & Moderado (2) & Leve (3) & Ninguno (4) \\
\hline \multirow{6}{*}{$\begin{array}{c}\text { Úlceras por } \\
\text { presión } \\
\text { (Escala de }^{\text {Braden) }}{ }^{53}\end{array}$} & $\begin{array}{l}\text { Percepción } \\
\text { sensorial }\end{array}$ & $\begin{array}{l}\text { Complemente } \\
\text { limitada }\end{array}$ & Muy limitada & $\begin{array}{l}\text { Ligeramente } \\
\text { limitada }\end{array}$ & Sin limitación \\
\hline & $\begin{array}{l}\text { Exposición a la } \\
\text { humedad }\end{array}$ & Siempre & A menudo & Ocasionalmente & Raramente \\
\hline & Actividad física & Encamado & En silla & $\begin{array}{l}\text { Deambula } \\
\text { ocasionalmente }\end{array}$ & $\begin{array}{l}\text { Deambula } \\
\text { frecuentemente }\end{array}$ \\
\hline & $\begin{array}{l}\text { Movilidad cambios } \\
\text { posturales }\end{array}$ & Inmóvil & Muy limitada & Levemente & Sin limitación \\
\hline & Nutrición & Muy pobre & $\begin{array}{l}\text { Probablemente } \\
\text { inadecuado }\end{array}$ & Adecuado & Excelente \\
\hline & $\begin{array}{l}\text { Cizallamiento y } \\
\text { roce }\end{array}$ & Presente & $\begin{array}{l}\text { Potencialmente } \\
\text { presente }\end{array}$ & $\begin{array}{l}\text { No existe problema } \\
\text { aparente }\end{array}$ & \\
\hline $\begin{array}{l}\text { Indicador } \\
\text { NOC }\end{array}$ & Parámetro a evaluar & Grave (1) & Moderado (2) & Leve (3) & Ninguno (4) \\
\hline \multirow{5}{*}{$\begin{array}{c}\text { Úlceras por } \\
\text { presión } \\
\text { (Escala de } \\
\text { Norton) }^{54}\end{array}$} & $\begin{array}{l}\text { Estado físico } \\
\text { general }\end{array}$ & Muy malo & Malo & Regular & Bueno \\
\hline & Estado mental & $\begin{array}{l}\text { Estuporoso o } \\
\text { comatoso }\end{array}$ & Confuso & Apático & Alerta \\
\hline & Movilidad & Inmóvil & Muy limitada & Disminuida & Total \\
\hline & Actividad & Encamado & Sentado & Camina con ayuda & Ambulante \\
\hline & Incontinencia & Urinaria y fecal & Urinaria o fecal & Ocasional & Ninguno \\
\hline
\end{tabular}

NOC: Nursing Outcomes Classification. 
Figura 1. Áreas potenciales de presión en la posición prono

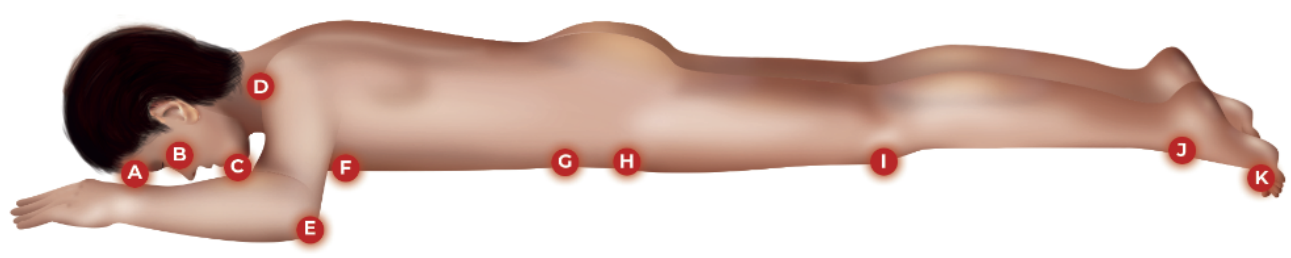
A. Frente
G. Genitales
B. Mejillas y Nariz
H. Borde anterior del hueso pélvico
C. Barbilla
I. Rodillas
D. Clavícula/Hombro
J. Dorso del pie
E. Codo
K. Dedos del pie
F. Pecho

Fuente: propia.

Figura 2. Guía del uso profiláctico de apósitos para disminuir la presión y prevenir las úlceras por presión

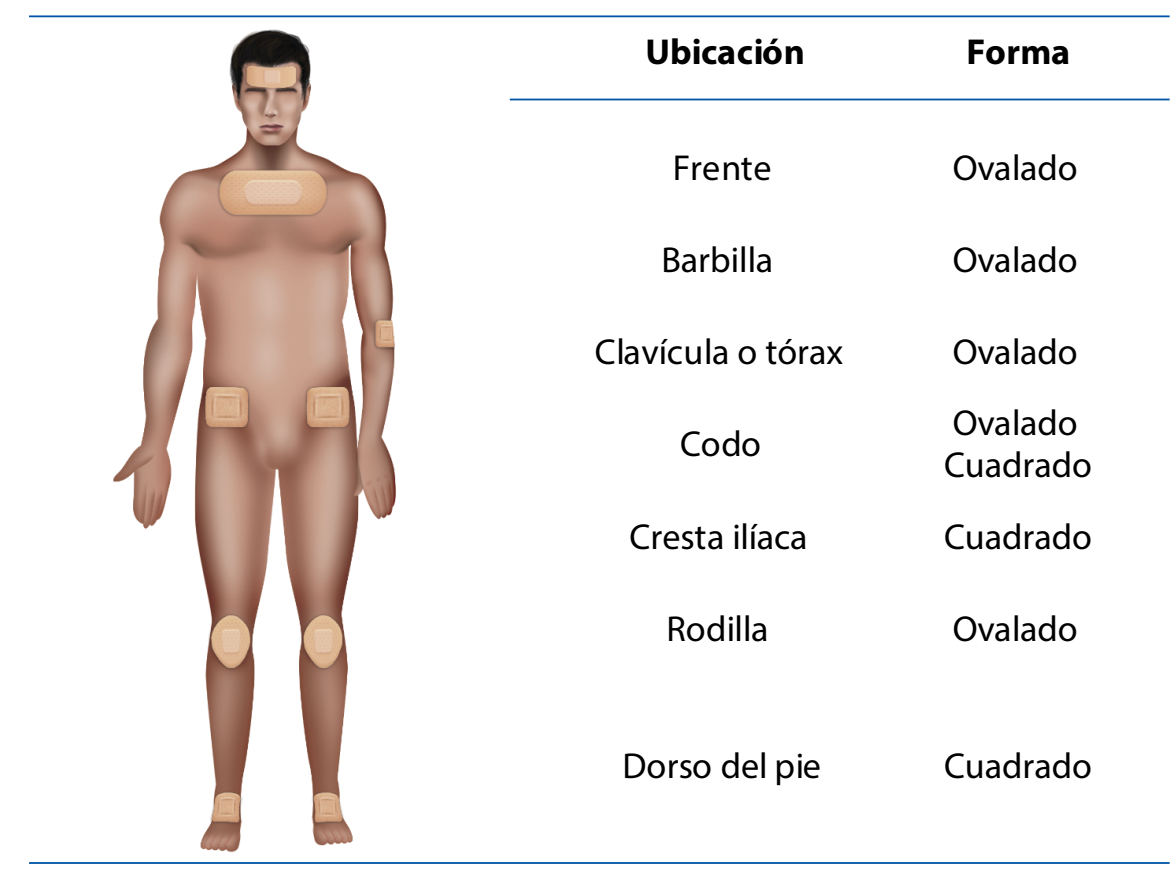

Fuente: propia.

Figura 3. Posición de natación, estilo libre

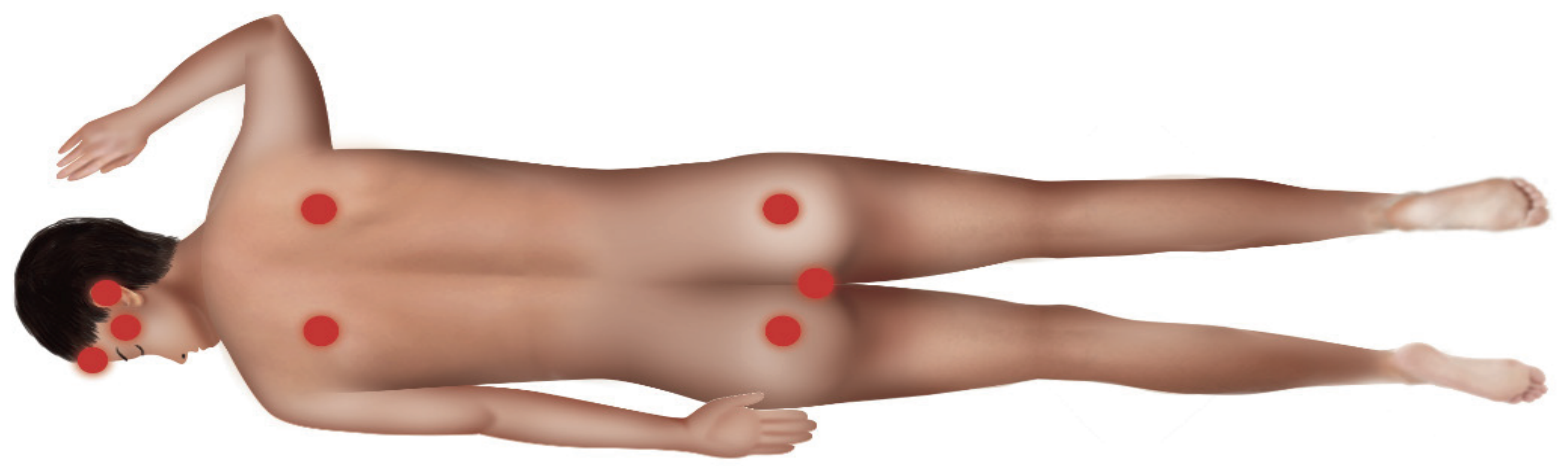

Fuente: propia. Los puntos de presión de la figura corresponden a la superficie del cuerpo que mira hacia el colchón, previamente descritos en la figura 1. 


\section{Consideraciones Finales}

Se requieren equipos de trabajo capacitadosy entrenados tanto para brindar los cuidados relacionados con la PP como para prevenir las UPP, debido a que lo anterior demanda un trabajo más intenso y es todo un desafío para el personal de enfermería. Existe una herramienta para demostrar la competencia de enfermería en la PP de los pacientes y evalúa cinco aspectos: 1) indica las condiciones para colocar al paciente en la PP; 2) declara

Se requieren equipos de trabajo capacitados y entrenados tanto para brindar los cuidados relacionados con la PP como para prevenir las UPP, debido a que lo anterior demanda un trabajo más intenso y es todo un desafío para el personal de enfermería.

las contraindicaciones para la PP; 3) prepara al paciente para la PP; 4) ayuda al paciente a colocarse en la PP y garantiza una posición anatómica correcta, y 5) evalúa la respuesta del paciente a la $\mathrm{PP}^{55}$, la cual podría ser útil a nivel clínico.

En resumen, la PP es una terapia coadyuvante recomendada para el manejo de pacientes con COVID-19 críticamente enfermos con manifestaciones de SDRA grave. Su uso se ha incrementado debido a los beneficios para mejorar la función pulmonar, sin embargo, también se ha asociado a eventos adversos como las UPP y otras complicaciones. Este artículo presenta recomendaciones basadas en la literatura científica para sustentar un plan de cuidados de enfermería dirigido a la implementación
Este artículo presenta recomendaciones basadas en la literatura científica para sustentar un plan de cuidados de enfermería dirigido a la implementación de actividades preventivas que reduzcan la frecuencia de las UPP secundarias a la PP en la enfermedad de COVID-19 de actividades preventivas que reduzcan la frecuencia de las UPP secundarias a la PP en la enfermedad de COVID-19, promoviendo el cuidado integral de calidad. Sin embargo, se debe tener precaución en la interpretación de estas recomendaciones dado que se realizó una revisión narrativa para sustentar el plan de cuidados y, debido al alcance de la misma no se evaluó la calidad metodológica de los artículos, por lo que se requiere de una búsqueda sistemática para definir su nivel de evidencia y grado de recomendación en la práctica clínica de enfermería.

Financiación: La elaboración de este artículo no requirió financiación.

Agradecimientos: Los autores agradecen a Juan Daniel Polanco Muñoz por el diseño de las imágenes presentadas en este artículo.

\section{Referencias}

1. Barker J, Koeckerling D, West R. A need for prone position CPR guidance for intubated and non-intubated patients during the COVID-19 pandemic. Resuscitation. 2020. 151:135-6. https://doi.org/10.1016/j.resuscitation.2020.04.029

2. Coppo A, Bellani G, Winterton D, Di Pierro M, Soria A, Faverio P, et al. Feasibility and physiological effects of prone positioning in non-intubated patients with acute respiratory failure due to COVID-19 (PRON-COVID): a prospective cohort study. Lancet Respir Med. 2020. 8(8):765-74. https://doi.org/10.1016/S2213-2600(20)30268-X

3. Bellani G, Laffey JG, Pham T, Madotto F, Fan E, Brochard L, et al. Noninvasive Ventilation of Patients with Acute Respiratory Distress Syndrome. Insights from the LUNG SAFE Study. Am J Respir Crit Care Med. 2017. 195(1):67-77. https://doi.org/10.1164/rccm.201606-1306OC

4. Guerin $\mathbf{C}$, Beuret $\mathbf{P}$, Constantin JM, Bellani G, Garcia-Olivares $\mathbf{P}$, Roca $\mathbf{O}$, et al. A prospective international observational prevalence study on prone positioning of ARDS patients: the APRONET (ARDS Prone Position Network) study. Intensive Care Med. 2018. 44(1):22-37. https://doi.org/10.1007/s00134-017-4996-5 
5. Barakat-Johnson M, Carey R, Coleman K, Counter K, Hocking K, Leong T, et al. Pressure injury prevention for COVID-19 patients in a prone position. Wound Practice and Research. 2020. 28(2):50-7. https://doi.org/10.33235/wpr.28.2.50-57

6. Settembre N, Maurice P, Paysant J, Theurel J, Claudon L, Kimmoun A, et al. The use of exoskeletons to help with prone positioning in the intensive care unit during COVID-19. Ann Phys Rehabil Med. 2020. 63(4):379-82. https://doi.org/10.1016/j.rehab.2020.05.004

7. Schub E, Pilgrim J. Patient positioning (critical care patients): prone. Nursing practice \& Skill. 2018. https://www.baccn.org/static/uploads/resources/NRCP_Prone_Position_ICU_pt.pdf

8. Ghelichkhani P, Esmaeili M. Prone Position in Management of COVID-19 Patients; a Commentary. Arch Acad Emerg Med. 2020. 8(1):e48. https://www.ncbi.nIm.nih.gov/pmc/articles/ PMC7158870/

9. Mora-Arteaga JA, Bernal-Ramirez OJ, Rodriguez SJ. The effects of prone position ventilation in patients with acute respiratory distress syndrome. A systematic review and metaanalysis. Med Intensiva. 2015. 39(6):359-72. https://doi.org/10.1016/j.medine.2014.11.004

10. Munshi L, Del Sorbo L, Adhikari NKJ, Hodgson CL, Wunsch H, Meade MO, et al. Prone Position for Acute Respiratory Distress Syndrome. A Systematic Review and Meta-Analysis. Ann Am Thorac Soc. 2017. 14(Supplement_4):S280-S8. https://doi.org/10.1513/AnnalsATS.201704-3430T

11.Stephen-Haynes J, Maries M. Pressure ulcers and the prone position. Br J Nurs. 2020. 29(12):S6. https://doi.org/10.12968/bjon.2020.29.12.S6

12.Bentley SK, lavicoli L, Cherkas D, Lane R, Wang E, Atienza M, et al. Guidance and Patient Instructions for Proning and Repositioning of Awake, Nonintubated COVID-19 Patients. Acad Emerg Med. 2020. https://doi.org/10.1111/acem.14067

13.Bamford P, Bentley A, Dean J, Whitmore D, Wilson-Baig N. ICS Guidance for Prone Positioning of the Conscious COVID Patient 2020. Intensive care society. https://emcrit.org/ wp-content/uploads/2020/04/2020-04-12-Guidance-for-conscious-proning.pdf

14.Albott CS, Wozniak JR, McGlinch BP, Wall MH, Gold BS, Vinogradov S. Battle Buddies: Rapid Deployment of a Psychological Resilience Intervention for Health Care Workers During the COVID-19 Pandemic. Anesth Analg. 2020. 131(1):43-54. https://doi.org/10.1213/ ANE.0000000000004912

15.Shearer SC, Parsa KM, Newark A, Peesay T, Walsh AR, Fernandez S, et al. Facial Pressure Injuries from Prone Positioning in the COVID-19 Era. Laryngoscope. 2021. https://doi. org/10.1002/lary.29374

16.Rodriguez-Huerta MD, Diez-Fernandez A, Rodriguez-Alonso MJ, Robles-Gonzalez M, Martin-Rodriguez M, Gonzalez-Garcia A. Nursing care and prevalence of adverse events in prone position: Characteristics of mechanically ventilated patients with severe SARS-CoV-2 pulmonary infection. Nurs Crit Care. 2021. https://doi.org/10.1111/nicc.12606

17.Ibarra G, Rivera A, Fernandez-Ibarburu B, Lorca-Garcia C, Garcia-Ruano A. Prone position pressure sores in the COVID-19 pandemic: The Madrid experience. J Plast Reconstr Aesthet Surg. 2020. https://doi.org/10.1016/j.bjps.2020.12.057

18.Capasso V, Cox J, Cuddigan J, Delmore B, Tescher A, Solmos S. Pressure injury prevention-pip tips for prone positioning. National pressure injury advisory panel. 2020 https:// cdn.ymaws.com/npiap.com/resource/resmgr/online_store/posters/npiap_pip_tips_-_proning_202.pdf

19.Peko L, Barakat-Johnson M, Gefen A. Protecting prone positioned patients from facial pressure ulcers using prophylactic dressings: A timely biomechanical analysis in the context of the COVID-19 pandemic. Int Wound J. 2020. https://doi.org/10.1111/iwj.13435

20.Kim RS, Mullins K. Preventing Facial Pressure Ulcers in Acute Respiratory Distress Syndrome (ARDS). J Wound Ostomy Continence Nurs. 2016. 43(4):427-9. https://doi.org/10.1097/ WON.0000000000000247 
21.Zingarelli EM, Ghiglione M, Pesce M, Orejuela I, Scarrone S, Panizza R. Facial Pressure UIcers in a COVID-19 50-year-old Female Intubated Patient. Indian J Plast Surg. 2020. 53(1):1446. https://doi.org/10.1055/s-0040-1710403

22.Perrillat A, Foletti JM, Lacagne AS, Guyot L, Graillon N. Facial pressure ulcers in COVID-19 patients undergoing prone positioning: How to prevent an underestimated epidemic?. J Stomatol Oral Maxillofac Surg. 2020. https://doi.org/10.1016/j.jormas.2020.06.008

23.Coleman S, Nixon J, Keen J, Wilson L, McGinnis E, Dealey C, et al. A new pressure ulcer conceptual framework. J Adv Nurs. 2014. 70(10):2222-34. https://doi.org/10.1111/jan.12405

24.Boyko TV, Longaker MT, Yang GP. Review of the Current Management of Pressure Ulcers. Adv Wound Care (New Rochelle). 2018. 7(2):57-67. https://doi.org/10.1089/wound.2016.0697

25.Gupta S, Hayek SS, Wang W, Chan L, Mathews KS, Melamed ML, et al. Factors Associated With Death in Critically III Patients With Coronavirus Disease 2019 in the US. JAMA Intern Med. 2020. https://doi.org/10.1001/jamainternmed.2020.3596

26.Taneri PE, Gomez-Ochoa SA, Llanaj E, Raguindin PF, Rojas LZ, Roa-Diaz ZM, et al. Anemia and iron metabolism in COVID-19: a systematic review and meta-analysis. Eur J Epidemiol. 2020. https://doi.org/10.1101/2020.06.04.20122267

27. Grasselli G, Zangrillo A, Zanella A, Antonelli M, Cabrini L, Castelli A, et al. Baseline Characteristics and Outcomes of 1591 Patients Infected With SARS-CoV-2 Admitted to ICUs of the Lombardy Region, Italy. JAMA. 2020. https://doi.org/10.1001/jama.2020.5394

28. Herramienta online para la consulta y diseño de planes de cuidados de enfermería. NNNConsult. Elsevier. 2015 [cited 2021 Abril08]. http://www.nnnconsult.com/

29. Moore Z, Patton D, Avsar P, McEvoy NL, Curley G, Budri A, et al. Prevention of pressure ulcers among individuals cared for in the prone position: lessons for the COVID-19 emergency. J Wound Care. 2020. 29(6):312-20. https://doi.org/10.12968/jowc.2020.29.6.312

30.Gefen A, Alves P, Ciprandi G, Coyer F, Milne CT, Ousey K, et al. Device-related pressure ulcers: SECURE prevention. J Wound Care. 2020. 29(Sup2a):S1-S52. https://doi.org/10.12968/ jowc.2020.29.Sup2a.S1

31.Park SH, Lee YS, Kwon YM. Predictive Validity of Pressure Ulcer Risk Assessment Tools for Elderly: A Meta-Analysis. West J Nurs Res. 2016. 38(4):459-83. https://doi. org/10.1177/0193945915602259

32.Park SH, Lee HS. Assessing Predictive Validity of Pressure Ulcer Risk Scales- A Systematic Review and Meta-Analysis. Iran J Public Health. 2016. 45(2):122-33. https://www.ncbi.nlm.nih. gov/pmc/articles/PMC4841867/

33. European Pressure Ulcer Advisory Panel NPIAPaPPPIA. Prevention and treatment of pressure ulcers/injuries: clinical practice guideline. The International Guideline. 2019. 3rd: http://www. internationalguideline.com

34.Girard R, Baboi L, Ayzac L, Richard JC, Guerin C, Proseva trial g. The impact of patient positioning on pressure ulcers in patients with severe ARDS: results from a multicentre randomised controlled trial on prone positioning. Intensive Care Med. 2014. 40(3):397-403. https://doi.org/10.1007/s00134-013-3188-1

35.Baktoft B. Nursing care of patients in the prone position. The World of Critical Care Nursing. 2001. 1(3):83-6. https://doi.org/10.1891/1748-6254.1.3.87

36.Lucchini A, Bambi S, Mattiussi E, Elli S, Villa L, Bondi H, et al. Prone Position in Acute Respiratory Distress Syndrome Patients: A Retrospective Analysis of Complications. Dimens Crit Care Nurs. 2020. 39(1):39-46. https://doi.org/10.1097/DCC.0000000000000393

37. Barakat-Johnson M, Lai M, Wand T, Li M, White K, Coyer F. The incidence and prevalence of medical device-related pressure ulcers in intensive care: a systematic review. J Wound Care. 2019. 28(8):512-21. https://doi.org/10.12968/jowc.2019.28.8.512

38.Black J, Alves P, Brindle CT, Dealey C, Santamaria N, Call E, et al. Use of wound dressings to enhance prevention of pressure ulcers caused by medical devices. Int Wound J. 2015. 12(3):322-7. https://doi.org/10.1111/iwj.12111 
39.Park KH, Kim KS. Effect of a structured skin care regimen on patients with fecal incontinence: a comparison cohort study. J Wound Ostomy Continence Nurs. 2014. 41(2):161-7. https://doi.org/10.1097/WON.0000000000000005

40.Avsar P, Karadag A. Efficacy and Cost-Effectiveness Analysis of Evidence-Based Nursing Interventions to Maintain Tissue Integrity to Prevent Pressure Ulcers and Incontinence-Associated Dermatitis. Worldviews Evid Based Nurs. 2018. 15(1):54-61.

https://doi.org/10.1111/wvn.12264

41.Otero DP, Dominguez DV, Fernandez LH, Magarino AS, Gonzalez VJ, Klepzing JV, et al. Preventing facial pressure ulcers in patients under non-invasive mechanical ventilation: a randomised control trial. J Wound Care. 2017. 26(3):128-36.

https://doi.org/10.12968/jowc.2017.26.3.128

42.Weng MH. The effect of protective treatment in reducing pressure ulcers for non-invasive ventilation patients. Intensive Crit Care Nurs. 2008. 24(5):295-9. https://doi.org/10.1016/j.iccn.2007.11.005

43.Prebio M, Katz-Papatheophilou E, HeindI W, Gelbmann H, Burghuber OC. Reduction of pressure sores during prone positioning of ventilated intensive care patients by the pronehead support system: a pilot study. Wien Klin Wochenschr. 2005. 117(3):98-105. https://doi.org/10.1007/s00508-004-0299-2

44.Katzengold R, Gefen A. What makes a good head positioner for preventing occipital pressure ulcers. Int Wound J. 2018. 15(2):243-9. https://doi.org/10.1111/iwj.12857

45.Levy A, Kopplin K, Gefen A. Device-related pressure ulcers from a biomechanical perspective. J Tissue Viability. 2017. 26(1):57-68. https://doi.org/10.1016/j.jtv.2016.02.002

46.Grisell M, Place HM. Face tissue pressure in prone positioning: a comparison of three face pillows while in the prone position for spinal surgery. Spine. 2008. 33(26):2938-41. https://doi.org/10.1097/BRS.0b013e31818b9029

47.Kumagai A, Ohno N, Miyati T, Okuwa M, Nakatani T, Sanada H, et al. Investigation of effects of urethane foam mattress hardness on skin and soft tissue deformation in the prone position using magnetic resonance imaging. J Tissue Viability. 2019. 28(1):14-20. https://doi.org/10.1016/j.jtv.2018.10.007

48. Roth S. Perioperative visual loss: what do we know, what can we do?. Br J Anaesth. 2009. 103 Suppl 1:i31-40. https://doi.org/10.1093/bja/aep295

49.Gefen A, Farid KJ, Shaywitz I. A review of deep tissue injury development, detection, and prevention: shear savvy. Ostomy Wound Manage. 2013. 59(2):26-35. https://pubmed.ncbi.nlm.nih.gov/23388395/

50.Shi C, Dumville JC, Cullum N. Support surfaces for pressure ulcer prevention: A network meta-analysis. PLoS One. 2018. 13(2):e0192707. https://doi.org/10.1371/journal.pone.0192707

51.Guerin C, Reignier J, Richard JC, Beuret P, Gacouin A, Boulain T, et al. Prone positioning in severe acute respiratory distress syndrome. N Engl J Med. 2013. 368(23):2159-68. https://doi.org/10.1056/NEJMoa1214103

52.Araujo MS, Santos M, Silva CJA, Menezes RMP, Feijao AR, Medeiros SM. Prone positioning as an emerging tool in the care provided to patients infected with COVID-19: a scoping review. Rev Lat Am Enfermagem. 2021. 29:e3397. https://doi.org/10.1590/1518-8345.4732.3397

53. Bergstrom N, Braden BJ, Laguzza A, Holman V. The Braden Scale for Predicting Pressure Sore Risk. Nurs Res. 1987. 36(4):205-10. https://doi.org/10.1097/00006199-198707000-00002

54.Norton D, McLaren R, Exton-Smith A. An investigation of geriatric nursing problems in hospital. London: Churchill Livingstone. Scientific Research. 1962. https://www.scirp.org/ (S(czeh2tfqyw2orz553k1w0r45))/reference/ReferencesPapers.aspx?ReferencelD=1191367

55.Drahnak DM, Custer N. Prone Positioning of Patients With Acute Respiratory Distress Syndrome. Crit Care Nurse. 2015. 35(6):29-37. https://doi.org/10.4037/ccn2015753 